\title{
A Case Report of Pediatric Kawasaki Disease and COVID-19 Association
}

\author{
Soroosh Salehabadi (iD ${ }^{1}$, Shima Shekari ${ }^{2,}{ }^{*}$, Shiva Shadani ${ }^{2}$ and Mohammad Shoja ${ }^{2}$ \\ ${ }^{1}$ Imam Hasan General Hospital Emergency Department, North Khorasan University of Medical Sciences, Bojnurd, Iran \\ ${ }^{2}$ Pediatric Department, North Khorasan University of Medical Sciences, Bojnurd, Iran \\ "Corresponding author: Pediatric Department, North Khorasan University of Medical Sciences, Bojnurd, Iran. Email: sh.shekari@nkums.ac.ir
}

Received 2020 July 14; Revised 2020 November 30; Accepted 2020 December 06.

\begin{abstract}
Introduction: As COVID-19 spreads rapidly all over the world and nations struggle to control it, the novel presentations of SARS$\mathrm{CoV}-2$ infection and its possible triggering role for other diseases in pediatrics concern clinicians in frontlines.

Case Presentation: We describe a 10-year-old child diagnosed with COVID-19 infection and concurrent Kawasaki disease. He presented with prolonged fever and conjunctivitis. His initial echocardiogram showed coronary artery dilation in RCA. He was treated with IVIG and aspirin as per guidelines and discharged 48 hours after the completion of IVIG and diminishing fever. His follow-up echocardiogram showed improvement in a two weeks' interval while he was quarantined in the meantime and showed no respiratory complications.

Conclusions: In conclusion, we think that there might be a correlation between COVID-19 infection and hyperinflammatory conditions, like Kawasaki disease. Further investigations are needed to enlighten the complications caused by COVID-19 infection, especially in pediatrics. In addition, we emphasize follow-up visits (in person or long-distance) in pediatrics presenting with inflammatory symptoms.

Keywords: Kawasaki Disease (KD), Coronavirus Disease 2019 (COVID-19), Severe Acute Respiratory Syndrome Coronavirus 2 (SARS-CoV-2), Multisystem Inflammatory Syndrome in Children (MIS-C), Intravenous Immune Globulin (IVIG), Coronary Artery Disease
\end{abstract}

\section{Introduction}

The widespread of novel Coronavirus Disease 2019 (COVID-19), caused by SARS-CoV-2 since January 2020 (1) in China, which then turned into a pandemic (2), has confronted us with a thought-provoking situation in the diagnosis and treatment of other diseases. The mild severity might disguise the early stages of other inflammatory diseases, some of which are harmless, like the common cold, and some cause morbidity or even mortality like Kawasaki Disease (KD). Epidemiologic data show a small proportion of pediatrics with positive COVID-19 in comparison with adults (3), accompanied by less severe symptoms (4). The fewer reports of pediatric cases of COVID-19 tied pediatricians' hands to come up with guidelines covering such issues.

In a case report, Jones et al. described a case of a sixmonth-old infant diagnosed with classic KD and COVID-19, who was treated as per guidelines with IVIG, aspirin, and 14-day quarantine at home (5). Licciardi et al. reported two cases of concurrent infection with COVID-19 and KD. They described these cases as SARS-CoV-2-induced Kawasaki-Like Hyperinflammatory (SCiKH) syndrome. They also sug- gested that intravenous corticosteroids are useful in the treatment of this situation (6). Rivera-Figueroa et al. reported a five-year-old child who was positive for COVID-19 and also met the criteria for incomplete KD. He was treated with IVIG and aspirin and quarantined for 14 days (7). In a case series published in May 2020, Riphagen et al. reported a cluster of hyperinflammatory shock in eight pediatric patients with features similar to KD shock syndrome, atypical $\mathrm{KD}$, and toxic shock syndrome (8).

Here, we describe a case of a pediatric patient of 10years-old diagnosed with KD and coinfection by COVID-19 with permission from parents.

\section{Case Presentation}

The patient was a 10-year-old, previously healthy, fully immunized boy. He had normal stature; his weight was 32 $\mathrm{kg}$ (between the 25 and 50 percentile), and his height was $147 \mathrm{~cm}$ (between the 75 and 90 percentile). First, he had a low-grade fever (101.3 F) and was diagnosed with the common cold, which was treated by conservative measures, such as acetaminophen and oral fluid replacement. After 
two days of persistent fever, the patient presented to the pediatrician with cervical lymphadenopathy. Cervical lymphadenopathy was described as the enlargement of tender, painful, and reddish left anterior chain cervical lymph nodes in the second zone. He was referred to a general hospital for further evaluation.

The patient presented with a fever of $102.2 \mathrm{~F}$ with partial improvement with antipyretics, and tender, painful, reddish, and enlarged left lateral side of the neck. The patient also described the signs of restlessness, decreased appetite, and painful joints in previous days. He also complained of nausea and a few episodes of non-projectile, non-bloody vomiting, without any other gastrointestinal signs and symptoms. In history-taking and examination, he did not have any signs and symptoms of cough, rhinorrhea, congestion, otitis, or toothache. He was admitted with a possible diagnosis of lymphadenitis.

The patient was admitted to the pediatric ward and treated with $300 \mathrm{mg}$ IV clindamycin antibiotic at six-hour intervals. The intravenous fluid replacement was conducted as 1,500 cc half-saline plus $15 \mathrm{cc}$ of $\mathrm{KCl} \mathrm{15 \%} \mathrm{for} 24$ hours. Besides, $325 \mathrm{mg}$ acetaminophen was administered every six hours to decrease fever and inflammation. Additional paraclinical studies and laboratory investigations were done, as well.

Ultrasonography of the abdomen and pelvis was intact, except for free fluid between intestinal loops. Soft tissue ultrasonography of the neck revealed multiple reactive lymph nodes in the left mandibular area of the neck; no evidence of abscess formation was seen in the study. Laboratory results showed the signs of inflammation with elevated ESR to 116 and three-plus-positive CRP. Mild to moderate leukocytosis with elevated white blood cells of 16.3 was detected with $82 \%$ neutrophils and $8 \%$ lymphocytes differentiation (Figure 1).

On the third day of hospitalization (the fifth day of sickness), the fever was persisting, and the signs of bilateral non-purulent conjunctivitis were noted (Figure 2). A cardiovascular consult was committed to rule out possible atypical KD coronary complications. Later that night, the PCR test results for COVID-19 virus RNA came back positive, and the patient was transferred to another hospital to be isolated for the same reason. Before beginning standard therapy for COVID-19, baseline ECG was conducted to examine specifically for QT intervals and a PPD test for possible TB infection, which was negative. The patient was on oral hydroxychloroquine 160 mg every 12 hours for the first day and $80 \mathrm{mg}$ every 12 hours for the rest.

Echocardiography showed mild dilation of coronary arteries. Besides, RCA was $4 \mathrm{~mm}$, and LMCA was $3.5 \mathrm{~mm}$. According to Boston's criteria for Z-score of coronary arteries in children, the Z-score for RCA was calculated as 3.66 and for LMCA as 0.94. The cardiologist suggested KD as the possible etiology of CA dilation and asked for follow-up echocardiography at two-week, six-week, and six-month intervals. He also suggested IVIG and long-term aspirin treatment.

The patient received standard treatment for KD. Intravenous IVIG $64 \mathrm{~g}$ was administered with a dose of 80 $\mathrm{mg} / \mathrm{kg} /$ day. The patient also received IVIG for 18 hours, and his vital signs were monitored constantly during infusion. No adverse reaction was found during IVIG therapy. Antibiotic therapy continued as before, and $640 \mathrm{mg}$ oral aspirin treatment continued for two weeks.

Lung and mediastinum HRCT without contrast was intact. The patient's liver enzymes were mildly elevated (AST: 64 and ALT: 194). The albumin level fell to 2.9, and CBC showed leukocytosis with improved absolute neutrophil counts from 1,304 to 2,368. Cardiac troponin I came back negative (Table 1 and Figure 1 ).

\begin{tabular}{lcc}
\hline Table 1. Lab Results During Hospitalization & \\
\hline Lab Data & Result & Normal Values \\
\hline Blood sugar & 92 & $70-120$ \\
\hline Urea & 24 & $17-43$ \\
\hline Cr & 0.76 & $0.7-1.4$ \\
\hline Calcium & 9.3 & $8.6-10.3$ \\
\hline Sodium & 135.5 & $135-148$ \\
\hline Potassium & 3.8 & $3.5-5.3$ \\
\hline Triglycerides & 217 & $50-150$ \\
\hline Cholesterols & 170 & $50-200$ \\
\hline LDH & 383 & $<746$ \\
\hline ALT & 194 & $<37$ \\
\hline AST & 64 & $<41$ \\
\hline Albumin & 2.9 & $3.5-5.2$ \\
\hline Troponin I & Negative & \\
\hline
\end{tabular}

On the sixth day of hospitalization and about 24 hours after IVIG treatment, the patient's fever improved, and he well-tolerated oral food intake. At the same time, diffuse rashes appeared on the trunk and extremities, which was in favor of KD (Figure 3). The next day's lab results showed approximately the same values of inflammatory markers (ESR:120 and CRP: three-plus positive). Platelets also raised from 376,000 to 502,000 in 48 hours.

The next day, the patient was discharged after seven days of hospitalization with improved signs and symptoms. He was prescribed low-dose aspirin (100 mg daily), as he was not feverish for two days. Two weeks later, the follow-up visit with the same cardiologist showed improvement of dilated CAs in echocardiography. Besides, 


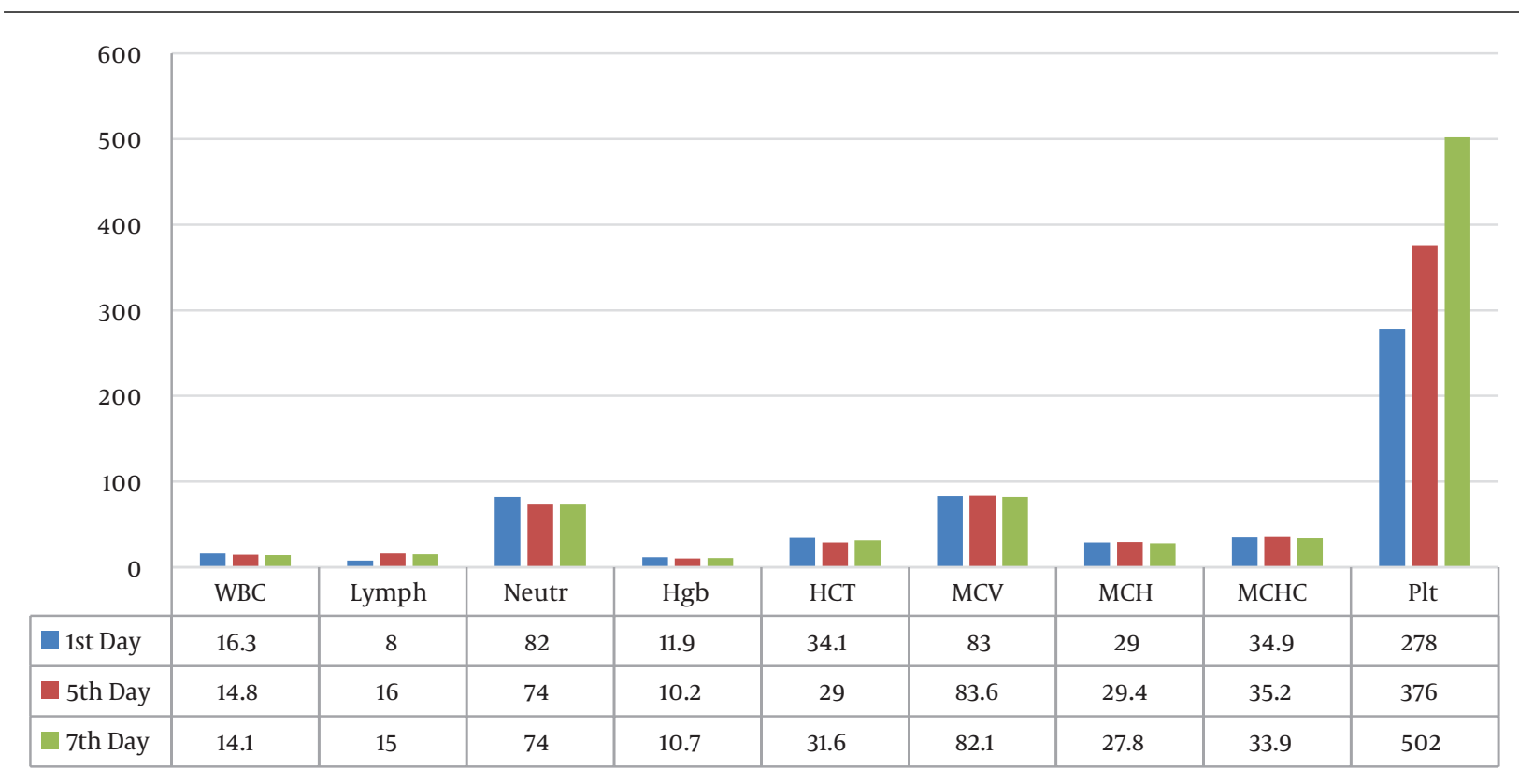

Figure 1. $\mathrm{CBC}$ results during hospitalization

RCA was measured as $3 \mathrm{~mm}$ (previously $4 \mathrm{~mm}$ ) and LMCA as $2.7 \mathrm{~mm}$ (previously $3 \mathrm{~mm}$ ). He was recommended to continue aspirin $80 \mathrm{mg}$ for the next six months.

\section{Discussion}

This is one of the first few papers in our region, which describes a case of pediatric KD with concurrent COVID-19 infection. It is not clear whether there is a correlation between these two or not. Typically, KD is more common during the first five years of life, but in our case, it occurred in the 10th year. This finding concerns us to be more objective in future cases of feverish pediatrics.

Kawasaki disease (also called mucocutaneous lymph node syndrome) is a common vasculitides of childhood, which rarely occurs in adults. Kawasaki disease is characterized by systemic inflammation, fever, and mucocutaneous involvement. The manifestations usually are not present at the same time. Thus, repeated examinations are essential for the timely diagnosis of KD, especially in the time of the COVID-19 pandemic, when rare diseases are not on the top list of differential diagnoses (9).

In our patient, the symptoms began with prolonged (about seven days) fever and cervical lymphadenopathy, which progressed to bilateral, nonexudative conjunctivitis, and coronary artery dilation. The mucocutaneous involvement was seen in this case as generalized rashes on the trunk and extremities. He did not complain of joint pain or movement disorders. Elevated ESR and CRP and dropped albumin levels were in favor of KD (Table 1).

According to WHO reports, COVID-19 infection has various presentations in the pediatric population, from completely asymptomatic to fever and upper respiratory involvement, like shortness of breath and cough. In severe cases, it could present as respiratory failure, shock, gastrointestinal symptoms, and coagulation dysfunction. In our patient, the course of infection was accompanied by a mild fever without respiratory symptoms such as shortness of breath. Investigations such as chest CT scan, pulse oximetry, and laboratory studies approved the clinical evaluations.

The recommended follow-up studies in KD suggest that the evaluation of coronary arteries should be performed in children regarding their coronary complication status. In the case of coronary artery dilation only, AHA recommends assessing the patient at two and six weeks of illness. If the dilation persisted, the assessments should be done every two to five years. If the dilation regressed to normal, the patient may be discharged after four weeks to 12 months. In our patient, the CA dilation regressed in the two-week follow-up study. We have planned to follow the patient in his next visit at six weeks of illness. We recommended that he takes aspirin until then.

Recently, the increasing reports of presentations like KD or toxic shock syndrome in children infected with COVID-19 have concerned health care providers about this 


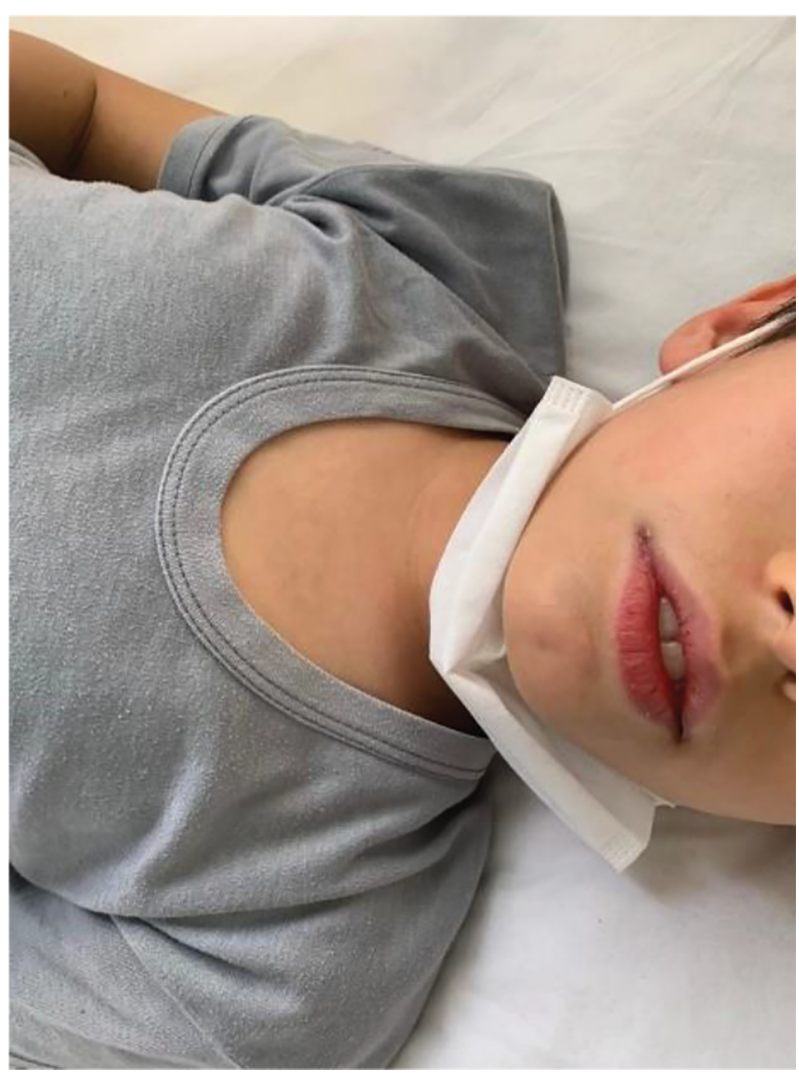

Figure 2. Red, dry, and cracked lips of the patient suggesting the underlying inflammatory condition

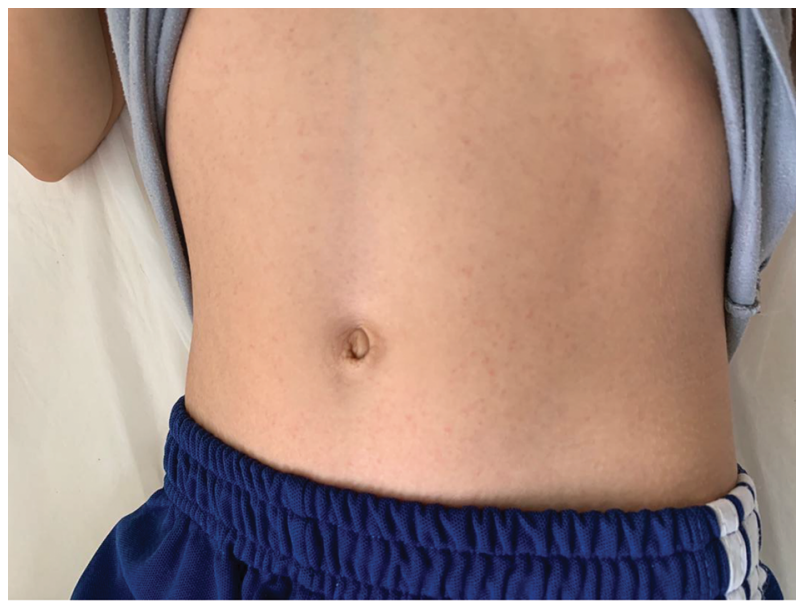

Figure 3. Diffuse truncal rash

new possible complication, termed Multisystem Inflammatory Syndrome in Children (MIS-C). The clinical manifestations include fever, gastrointestinal symptoms, con- junctivitis, and mucocutaneous involvement. The respiratory complications are uncommon, and there is no prominent feature in most cases.

In available case reports, the criteria for complete KD was met in 50 to $64 \%$ of cases. Our patient could be classified as MIS-C according to his signs and symptoms and advanced evaluations. We recommend further studies to clarify this syndrome prognosis and possible treatment approaches.

\section{Acknowledgments}

We thank the patient and his family for their cooperation and kindness to share this case and images. In addition, we thank all the staff of the pediatric department who contributed to the treatment of the patient discussed in this case report.

\section{Footnotes}

Authors' Contribution: Study concept and design: S. S.; Data collection: S. S. and M.S.; Drafting of the manuscript: S. S. and S. S.

Conflict of Interests: All authors have no conflict of interest to declare.

Funding/Support: All authors did not receive any funding/support.

Informed Consent: Informed consent was obtained, both verbally and in writing, from the parents, and their cooperation during the follow-up period approves this, as well.

\section{References}

1. WHO. Disease Outbreak News. Geneva, Switzerland: World Health Organization; 2020. Available from: https://www.who.int/csr/don/en/.

2. WHO. WHO Director-General's opening remarks at the media briefing on COVID-19 - 11 March 2020. Geneva, Switzerland: World Health Organization; 2020. Available from: https://www.who.int/directorgeneral/speeches/detail/who-director-general-s-opening-remarksat-the-media-briefing-on-covid-19---11-march-2020.

3. Cdc Covid- Response Team. Coronavirus disease 2019 in children United States, February 12-April 2, 2020. MMWR Morb Mortal Wkly Rep. 2020;69(14):422-6. doi: 10.15585/mmwr.mm6914e4. [PubMed: 32271728]. [PubMed Central: PMC7147903].

4. Tagarro A, Epalza C, Santos M, Sanz-Santaeufemia FJ, Otheo E, Moraleda C, et al. Screening and severity of coronavirus disease 2019 (COVID-19) in children in Madrid, Spain. JAMA Pediatr. 2020. doi: 10.1001/jamapediatrics.2020.1346. [PubMed:32267485]. [PubMed Central: PMC7142799].

5. Jones VG, Mills M, Suarez D, Hogan CA, Yeh D, Segal JB, et al. Covid19 and Kawasaki disease: Novel virus and novel case. Hosp Pediatr. 2020;10(6):537-40.doi:10.1542/hpeds.2020-0123. [PubMed: 32265235]. 
6. Licciardi F, Pruccoli G, Denina M, Parodi E, Taglietto M, Rosati S, et al. Sars-cov-2-induced Kawasaki-like hyperinflammatory syndrome: A novel covid phenotype in children. Pediatrics. 2020;146(2). doi: 10.1542/peds.2020-1711. [PubMed: 32439816].

7. Rivera-Figueroa EI, Santos R, Simpson S, Garg P. Incomplete Kawasaki disease in a child with COVID-19. Indian Pediatr. 2020;57(7):680-1. [PubMed: 32393680]. [PubMed Central: PMC7387257].

8. Riphagen S, Gomez X, Gonzalez-Martinez C, Wilkinson N, Theocharis
P. Hyperinflammatory shock in children during COVID-19 pandemic. Lancet. 2020;395(10237):1607-8. doi: 10.1016/S0140-6736(20)31094-1. [PubMed: 32386565]. [PubMed Central: PMC7204765].

9. McCrindle BW, Rowley AH, Newburger JW, Burns JC, Bolger AF, Gewitz M, et al. Diagnosis, treatment, and long-term management of Kawasaki disease: Ascientific statement for health professionals from the American heart association. Circulation. 2017;135(17):e927-99. doi: 10.1161/CIR.0000000000000484. [PubMed: 28356445]. 